\title{
Kein Vorteil durch umfassendere Betreuung
}

\author{
Wenn Intensivpatienten langfristig lebenserhaltende \\ Maßnahmen benötigen, stehen die Angehörigen unter einer \\ schweren emotionalen Belastung. In solchen Situationen \\ wächst der Wunsch nach einer stärker „sprechenden Medizin“. \\ In einer randomisierten Studie wurde nun ein neues \\ Betreuungskonzept untersucht. \\ JAMA 2016; 316: 51-62
}

Insgesamt 256 über 21-jährige Patienten und 365 Angehörige nahmen an der Studie teil. Studieneinschlusskriterien für die Patienten waren u.a. eine mindestens 7-tätige, nicht unterbrochene mechanische Beatmung und die fehlende Aussicht auf eine Entwöhnung in den nächsten 3 Tagen. Alle Angehörigen mit Entscheidungsvollmachten erhielten eine Informationsbroschüre über chronische lebensbedrohliche Krankheitssituationen. Die Mitarbeiter der Intensivstation standen für Besprechungen mit der Familie zu Verfügung.

Zusätzlich zur Standardbetreuung erfolgten in der Interventionsgruppe 2 strukturierte Treffen mit u.a. Palliativspezialisten, speziell ausgebildeten Krankenpflegerinnen, Sozialarbeitern und Geistlichen. Das erste Treffen fand am 7. Beatmungstag statt, wenn häufig eine Tracheostomie erwogen wird und das zweite frühestens 10

Tage später. Gesprächsinhalte waren

- medizinische Schulung (,information"),

- die Gefühle der Angehörigen und

- die Einbeziehung der bekannten oder vermuteten Patientenwünsche („support“).
Verglichen mit der Kontrollgruppe verfehlte die Intervention die Endpunkte nach 3 Monaten. Es ergab sich

- kein signifikanter Unterschied für Ängste und Depressionen ( $\mathrm{p}=0,34)$ sowie

- häufiger posttraumatische Belastungsstörungen $(p=0,0495)$

Bei Angehörigen mit intensiver Betreuung und posttraumatischer Belastungsstörung traten die typischen Symptome „Vermeidung“ und „Übererregbarkeit“ öfter auf. Für „Wiedererleben“ in Form von Flashbacks und Alpträumen bestand kein deutlicher Gruppenunterschied. Ein indirekt erzielter Vorteil für die Patienten konnte nicht bestätigt werden. Die Intervention beeinflusste das 90-Tage-Überleben, die Beatmungsdauer, Krankenhaustage und Medikation nicht wesentlich.

\section{Fazit}

Die ausgebliebene Entlastung der Familienangehörigen kann auf die hohe Qualität der Standardbetreuung oder aber auf die begrenzte Anzahl und Kontinuität der Interventionen zurückzuführen sein, so die Autoren.

\section{Dr. Susanne Krome, Melle}

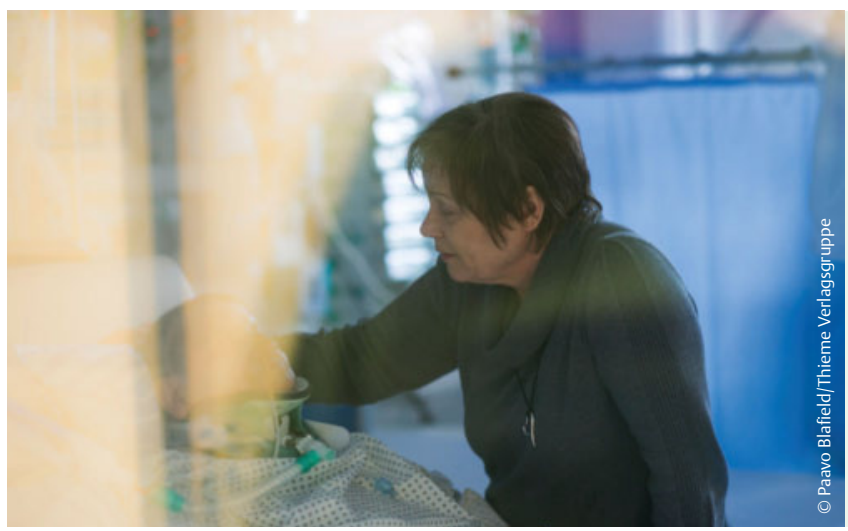

Ausschreibung

\section{Forschungspreise des Lungenfibrose e.V.}

Der Lungenfibrose e.V. schreibt anlässlich des 58. Kongresses der Deutschen Gesellschaft für Pneumologie und Beatmungsmedizin (DGP) im kommenden Jahr 3 Preise auf dem Gebiet der interstitiellen Lungenerkrankungen (ILD), insbesondere zur idiopathischen Lungenfibrose (IPF) aus.

Erstmalig wird im kommenden Jahr ein Preis zur Förderung des wissenschaftlichen Nachwuchses (Altersgrenze 40 Jahre) für das beste beim DGP-Kongress eingereichte wissenschaftliche Abstract zum Thema ILD oder IPF, der mit $3000 €$ dotiert ist, vergeben. Zudem wird zum ersten Mal ein Preis für die beste Fortbildung im Bereich interstitieller und seltener Lungenerkrankungen vergeben. Hiermit sollen ausdrücklich Bemühungen unterstützt werden, die sich um eine verbesserte Aufmerksamkeit und ein besseres Verständnis dieses Erkrankungsfeldes bemühen und hierzu versuchen, viele Kollegen zu erreichen. Hierfür wird ein Preisgeld von $1000 €$ ausgeschrieben. Darüber hinaus wird erstmalig ein Preis, der mit ebenfalls $1000 €$ dotiert ist, für die beste wissenschaftliche Arbeit aus Deutschland im Bereich interstitieller Lungenerkrankungen im Jahr 2016 prämiert.

Die aktuellen Arbeiten für den Nachwuchspreis und die beste wissenschaftliche Publikation dürfen zu keinem anderen Preis eingereicht sein. Die Preise werden jeweils anlässlich des DGP-Kongresses 2017 in Stuttgart vergeben.

Die Bewerber werden gebeten, ihre Bewerbung, die auch einen Lebenslauf und eine Publikationsliste enthalten sollte bis zum 31. Januar 2017 in PDF-Format (max. $5 \mathrm{MB}$ ) an die 1. Vositzende des Lungenfibrose e.V. Dagmar Kauschka (E-Mail: d.kauschka@lungenfibrose.de) zu schicken. Die Bewertung der eingereichten Bewerbungen erfolgt über den wissenschaftlichen Beirat der Lungenfibrose e.V. und berücksichtigt die Kriterien bei Preis 1 und 3 hinsichtlich Originalität, Methodologische Qualität und Klinische bzw. wissenschaflicher und edukativer Qualität.

Nach einer Mitteilung des Lungenfibrose e. V., Essen 